\title{
Protée
}

\section{Mécaniques de l’imaginaire}

\section{Guido Ferraro}

Volume 29, numéro 1, 2001

La société des objets. Problèmes d'interobjectivité

URI : https://id.erudit.org/iderudit/030614ar

DOI : https://doi.org/10.7202/030614ar

Aller au sommaire du numéro

\section{Éditeur(s)}

Département des arts et lettres - Université du Québec à Chicoutimi

\section{ISSN}

0300-3523 (imprimé)

1708-2307 (numérique)

Découvrir la revue

\section{Citer cet article}

Ferraro, G. (2001). Mécaniques de l'imaginaire. Protée, 29(1), 37-47. https://doi.org/10.7202/030614ar

\section{Résumé de l'article}

Les derniers développements technologiques sont en train de mettre certains genres d'objets - « bots » et robots - à même de communiquer et d'interagir entre eux en manifestant un degré toujours plus élevé d'autonomie. Toutefois, ce que nous avons tendance à saisir substantiellement comme des objets devrait, d'un point de vue sémiotique, être repensé plutôt en termes d'entités purement relationnelles, corrélées soit à d'autres " objets ", soit à des sujets. De même, si les objets sont envisagés le plus couramment comme des indicateurs d'identité sociale à lire par les autres sujets, ici au contraire on voit les objets-robots se conduire comme des lecteurs prompts à modéliser nos comportements, à l'attention d'autres robots. Des bots à notre service, on passe alors aux robots en société.
Ce document est protégé par la loi sur le droit d'auteur. L'utilisation des services d'Érudit (y compris la reproduction) est assujettie à sa politique d'utilisation que vous pouvez consulter en ligne.

https://apropos.erudit.org/fr/usagers/politique-dutilisation/ 


\section{MÉCANIQ UES DE L'IMAG IN AIRE}

GUIDO FERRARO

Dans la première partie du film de Jean-Pierre Jeunet, Alien Resurrection - dont le scénario est riche en références symboliques concernant le rapport «SujetObjet»-, on assiste à une dramatique interaction entre deux objets de forme humaine, dont le premier est programmé (sur la base de principes "politically correct») pour détruire le second qui représente un grave danger pour l'humanité. Le premier objet, appelé Call, est une machine créée par des robots et a la forme d'une jeune femme. Le second, nommé tout simplement par un numéro, Numéro 8 , a été fabriqué en laboratoire par des savants avides et sans scrupules. Ceux-ci ont reproduit, en la dupliquant, une femme nommée Ripley - morte deux siècles auparavant-pour lui permettre de mener jusqu'au bout sa grossesse et recréer ainsi une espèce éteinte d'extraterrestres.

En fait, Call rate sa mission, puisqu'elle n'élimine pas Numéro 8: celle-ci a bel et bien donné naissance à une race de monstres. Et Call s'aperçoit en outre avec horreur que la chaîne des dépendances logiques et temporelles a été altérée, car la «fille» née de Numéro 8 a rétroagi sur le patrimoine biologique de sa mère (ce bouleversement de la ligne de détermination est, en effet, très marquant dans cette histoire).

Par la suite, les deux «femmes-choses» découvrent qu'elles peuvent interagir de façon très créative, puisque chacune d'elles est l'envers de l'autre. La première, automate né d'un processus de programmation consciente, se charge d'effacer ses propres antécédents, en éliminant le «Père», entité à la fois métaphysique et technologique. L'autre, vierge mère produite en laboratoire, se charge au contraire de détruire sa descendance, jusqu'au monstrueux petit-fils mi-extra-terrestre mihumain (qu'elle tue de façon significative en utilisant son propre sang).

Ayant ainsi échappé à leur statut d'objets insérés dans une logique causale, les deux héroïnes, fascinées et "étrangères", tournent enfin les yeux pour la première fois vers la planète Terre et vers un avenir imprévisible. Elles peuvent enfin se poser comme d'authentiques sujets. Car c'est cela justement qui nous rend différents des objets, dans la représentation, même naïve, que nous, les humains, voulons en donner: nous aimons nous penser comme des entités disjointes de leurs antécédents comme de leurs conséquents, effrayés que nous sommes et fascinés aussi par un 
environnement auquel nous nous sentons dans un certain sens étrangers, prêts à des programmes de transformations virtuelles, et à un avenir qui, par définition, doit nous rester inconnu. Les machines interagissent entre elles en se traitant comme des objets insérés dans des chaînes de détermination; les êtres humains, au contraire, interagissent entre eux en se voyant comme des entités qui conçoivent des projets, créent un imaginaire, fondent leur agir sur une insondable base pathémique d'attractions et de craintes.

\section{LES ROBOTS À NOTRE SERVICE}

Je vais présenter ici quelques réflexions sémiotiques sur certains objets qui, à cause de récents développements technologiques, ont acquis la capacité de communiquer et d'interagir entre eux, accédant ainsi à un niveau toujours plus grand d'autonomie: des réfrigérateurs qui prennent l'initiative de faire le marché par l'intermédiaire d'un service automatisé d'e-commerce, des fours à micro-ondes qui consultent des bibliothèques gastronomiques en ligne et qui composent des menus spécifiquement étudiés pour nos invités, des voitures qui découvrent par ellesmêmes leurs défauts et appellent le bureau d'assistance pour prendre rendez-vous, après avoir, bien sûr, consulté notre agenda électronique afin de choisir le jour le plus approprié. Une attention particulière doit être accordée à ce qu'on appelle les bots, c'est-à-dire ces robots «logiciels» qui, comme certains le prévoient, vont jouer un rôle de plus en plus important dans notre vie en qualité d' «agents", c'est-à-dire d'assistants personnels.

Tout cela nous renvoie à cet objectif évident, sousjacent à toute philosophie de l'automation, qui consiste à décharger les sujets humains des tâches répétitives et mécaniques, de façon à ce qu'ils puissent se consacrer à des activités plus enrichissantes - à caractère plus complexe ou plus créatif.

Une recherche sur ces objets peut fournir, bien sûr, des éléments utiles pour mieux comprendre ce qu'est le «Sujet» (élément trop mal défini dans la sémiotique courante), mais elle peut surtout contribuer à la construction de modèles théoriques efficaces, capables de rendre compte à la fois d'une subjectivité répartie dans la société et des interactions que cette subjectivité va entraîner.

Le mot «bot», dérivé du terme plus connu «robot", est employé pour différencier les versions uniquement logicielles de l'idée traditionnelle du "fidèle serviteur mécanique». Beaucoup de ces créatures logicielles sont perçues comme des expériences d'intelligence artificielle en même temps que comme des solutions à des problèmes concrets. Dans le cas le plus banal, nous l'avons dit, un bot décharge son "propriétaire» de plusieurs tâches répétitives et ennuyeuses; dans les cas les plus complexes, il permet la réalisation d'opérations autrement impossibles ou pratiquement inaccessibles à l'agir humain, lent et discontinu. Ici particulièrement, il conviendrait de faire remarquer que ces bots sont des prolongements, des prothèses de certains aspects de la pensée et de l'action humaines.

Dans un ouvrage aux tendances vaguement darwiniennes, Andrew Leonard propose cette définition:

[...] un bot est un logiciel qui se présente comme intelligent, et qui se caractérise par le fait d'être capable d'autonomie et d'avoir sa personnalité propre; bien sûr, en plus mais pas toujours, il rend service. ${ }^{1}$

Il faut noter la référence à l'autonomie, qui suppose une prédisposition du bot à agir, non pas certes audelà de la volonté de son créateur (même si cela pourrait arriver, comme c'est le cas de tout logiciel ou de tout appareil susceptible de défaillances), mais en dehors de sa présence et, par conséquent, de son contrôle direct. Cela implique deux éléments importants pour notre discours: 1 . ces artefacts doivent avoir la capacité de garder dans le temps des attitudes programmées; 2 . ils doivent pouvoir s'activer à certaines conditions, c'est-à-dire savoir répondre aux changements de l'environnement dans lequel ils opèrent, ou encore se prêter à des interactions en présence d'autres sujets et objets actifs.

Évidemment, l'attribution d'une personnalité au bot est aussi un trait important à noter. Bien que cet 
aspect ne soit pas toujours présent chez la plupart des bots, on peut dire cependant qu'il s'agit autant d'un trait qui entre dans l'évolution de ces créatures logicielles que d'un trait qui devient évident chez ceux qu'on peut considérer comme des exemples types. Parmi les bots les plus connus, il y a en effet des automates créés pour soutenir une conversation en ligne. Eliza, confinée à des propos élémentaires (son apparition au MIT date de 1966), et surtout Julia - douée d'une remarquable habileté à établir des rapports thématiques - ont été de célèbres "chatterbots». Il est évident que ces bots sont conçus pour s'entretenir avec des humains, et qu'on n'a pas prévu pour eux des rapports imprévisibles avec d'autres objets.

Il est quand même évident qu'on est en présence d'un procès d'anthropomorphisation, analogue au processus qui sous-tend la construction de personnages, ou "acteurs", comme ceux qui agissent dans une structure narrative. Mais à la différence de ceux-ci, les bots ne sont pas seulement des êtres purement imaginaires: ils sont en mesure d'intervenir concrètement dans notre vie.

Si le lecteur pense que nous sommes en train de parler d'entités qui ne peuvent servir qu'à d'extravagants exercices théoriques, il faut lui rappeler que les technologies qui produisent ces entités font l'objet de formidables investissements, et qu'on peut être sûr qu'elles occuperont une très grande place dans l'avenir. À ce propos, rappelons que le créateur de Julia, Michael Mauldin, a conçu par la suite le célèbre spider de Lycos, l'un des robots parmi les mieux connus et les plus rentables, consacrés à l'indexation des pages Internet. C'est, en effet, Internet qui offre à ces robots leur lieu de travail le plus remarquable: ils peuvent parcourir sans arrêt l'océan intertextuel du grand réseau et répertorier ses contenus de façon rapide et exhaustive (de manière un peu naïve il est vrai), ce qui n'est pas à la portée des êtres humains.

Parce qu'il faut bien ajouter que si, évidemment, tous les bots agissent par l'intermédiaire d'un ordinateur, leur «milieu naturel» est celui du réseau, où ils peuvent exercer des activités comme la collecte d'informations, la recherche d'un document ou des coordonnées d'une personne, etc. On peut par exemple leur confier des tâches compliquées - comme le tri du courrier électronique reçu ou celui des messages d'un groupe de discussion selon tel ou tel critère. Et bien sûr un bot peut aussi réserver, acheter, payer, ou encore se mettre à la recherche de clients, présenter des catalogues de produits ou proposer des services particuliers à ceux qui sont susceptibles de s'y intéresser. Le fait que les bots peuvent occuper une place sur l'un et l'autre front de l'action commerciale nous fait bien voir comment pourra devenir de plus en plus fréquente l'éventualité qu'un bot, dans un régime de pleine interobjectivité, puisse faire face à un autre bot, activant ainsi des mécanismes, souvent complexes, de négociation.

On peut donner en ce sens autant d'exemples d'harmonieuse collaboration que d'âpre conflit. Notre bot personnel peut efficacement interagir avec les automates d'une entreprise de livraison pour vérifier si un colis que nous avons envoyé est sur la bonne route, et nous prévenir du moment où il arrivera à destination. Par ailleurs, un cas typique de conflit est illustré par ce qui se passe dans des groupes de discussion sur Internet, où des perturbations sont provoquées par certains bots indésirables, capables de nuire sérieusement au déroulement des interactions entre les participants. Contre ces bots, des bots modérateurs vont agir en s'efforçant de maintenir les échanges dans les limites et les règles prévues, en interdisant même l'accès au groupe à des interlocuteurs considérés comme étant de nature non humaine, ou bien en effaçant automatiquement les messages qui ne suivent pas les modèles textuels prévus (on parle dans ce cas de "cancelbots»). Nous avons ici affaire à des objets qui parlent à d'autres objets, des objets qui se donnent et qui se coupent la parole, des objets qui sanctionnent le comportement d'autres objets et qui les «tuent», en les excluant du groupe, sur le plan de la communication. Ce sont là des réalités qui nous deviennent peu à peu familières. Il est évident qu'il s'agit là de cas semblables, du point de vue conceptuel, 
à celui du réfrigérateur qui entretient des relations chaleureuses avec l'automate vendeur du supermarché, ou à celui du four à micro-ondes qui fréquente des bots gastronomes ou des bots bibliothécaires pour découvrir de nouvelles recettes à nous proposer. Mine de rien, tout un univers quasi surréel d'objets commence à exister: des objets se rencontrent, se mesurent et s'accordent, dans le seul but d'obtenir pour nous ce qu'il y a de mieux, de nous décharger des soucis quotidiens, de nous dorloter mieux qu'une vieille nounou.

En regard des emplois que pourraient occuper couramment les bots, l'idée la plus répandue (cette idée est soutenue notamment par l'incontournable Nicholas Negroponte) est celle qui voit dans les bots des assistants personnels (peut-être que dans cette perception l'image traditionnelle du robot, populaire et naïve, a son poids). Cette tendance, associée au fait qu'on pense confier à ces objets des missions à exercer surtout sur le réseau télématique, a mené, pour désigner ces bots, à l'introduction du terme plus spécifique d'«agent» - dont les valeurs, du point de vue sémiotique, peuvent aisément être perçues.

En tout cas, il est évident que les bots agissent fondamentalement sur des faits de nature communicationnelle (même si l'on prend en compte qu'Internet a tendance à changer profondément certaines de nos idées courantes sur la "communication»). Et cette dimension est sûrement importante dans une perspective de recherches qui examinent les relations des objets entre eux.

On pourrait dire en bref que ces nouvelles réalités semblent être caractérisées par au moins trois éléments. 1. Il s'agit de réalités logicielles, conçues pour interagir avec d'autres réalités du même genre. 2. Elles opèrent dans le milieu spécifique du réseau télématique, donc dans un univers bâti avec des matériaux de nature d'abord communicative. 3. Leur principale raison d'être consiste à remplacer les êtres humains dans des tâches dont l'étendue et les particularités restent encore à définir.

Nous allons examiner de près chacun de ces trois éléments.
OBJETS LOGICIELS ET COMPOSITIONS MULTIMÉDIAS

Il ne s'agit pas ici de soulever les questions relatives aux modes de réalisation des créatures logicielles - d'ailleurs je ne serais pas en mesure de le faire. Cependant, on ne saurait se passer des rapports importants, du point de vue théorique, entre certains aspects du fonctionnement de ces automates et agents et la logique qui préside à la production industrielle des logiciels. La logique actuelle n'est plus, en effet, fondée sur l'écriture linéaire d'un "code», composé d'un certain nombre d'instructions à exécuter, mais sur la connexion opérationnelle d'un ensemble d'objets (c'est le terme technique employé), dont chacun encapsule en soi son propre code, en même temps qu'il rend ses capacités accessibles aux autres objets.

Cela veut dire que ce que nous voyons comme une unité fonctionnelle - notre logiciel d'écriture, par exemple - agit en réalité comme un ensemble constitué de plusieurs «objets» (des dizaines ou des centaines d'objets différents), où chacun remplit des fonctions spécifiques et peut faire appel à l'un ou à l'autre des "objets» dont il est solidaire pour obtenir un service quelconque. Aucun de ces objets ne «connaît", si l'on peut employer ce terme, la logique qui régit les opérations effectuées par les autres objets, mais il «sait» comment les appeler, ce qu'il peut leur demander et quel résultat il peut en tirer. En outre, le rendement global du système dépend du fait que les objets produits par une entreprise, et qui font partie d'un certain logiciel, sont capables de reconnaître les objets créés par d'autres producteurs et insérés dans d'autres logiciels, de dialoguer avec eux et de bénéficier d'au moins quelques-uns des services qu'ils peuvent offrir. Bref, il semble que nous soyons en présence d'une sorte de société des objets.

La capacité des objets à se connecter entre eux, de manière à rendre possible une coopération mutuelle, apparaît comme fondamentale. Cette forme d'interobjectivité est donc liée à l'évolution même des modèles de la production industrielle. Il ne faut pas oublier qu'une des raisons essentielles qui motivent cette orientation est qu'elle permet d'éviter l'inutile 
duplication de fonctions analogues dans différents objets. C'est le cas, par exemple, quand il s'agit d'utiliser un même écran domestique pour visualiser les programmes de télévision, les pages Internet, les photographies de vacances, les jeux vidéo et les films: pour utiliser cet écran unique, il naît un appareil permettant de lire les films sur DVD ainsi que les CD musicaux, les CD-ROM, les photos-CD, ainsi de suite. L'économie qu'on réalise en évitant de telles duplications est évidente, de même que la tendance à mettre au rancart les objets mono-fonctionnels traditionnels (le téléviseur, la chaîne hi-fi, et pourquoi pas l'ordinateur...) en faveur d'objets dont l'identité sera moins précise et le caractère moins individualiste, des objets qui seront dotés d'une nature modulaire, et donc conçus dès le départ pour interagir entre eux. Il ne faut donc pas oublier que la composition interobjective peut se fonder sur d'importantes motivations d'ordre économique (une réflexion qui nous est d'ailleurs familière grâce aux nombreuses études linguistiques et sémiotiques consacrées aux perspectives componentielles).

Dans le même sens, on peut aussi penser à la très prochaine sortie de scène du téléphone cellulaire, remplacé par des agrégats multifonctionnels qui vont rassembler des modules tenant aussi bien de l'appareil photographique, du magnétophone, de l'ordinateur, du téléviseur, etc. Réfrigérateurs et fours de dernière génération constituent d'ailleurs, eux aussi, des réalités composites qui unissent aux composants traditionnels d'autres nouveaux modules - sous le contrôle, bien sûr, d'un agent, d'un bot.

Ce processus de décomposition en modules bouleverse les systèmes de communication auxquels on était habitué, posant les bases de ce qu'on appelle la "révolution multimédia». De la même façon, les programmes de télévision se fragmentent en de multiples modules qui peuvent être combinés de plusieurs manières, créant ainsi des formes toujours plus hybrides. Qui plus est, Internet nous propose des architectures de communication capables d'enchaîner des modules provenant de l'univers de la télévision, du téléphone, du journal et du cinéma, du centre commercial et de la salle d'attente. Des éléments sortis de différents médias sont liés entre eux, de manière à composer des ambiances de communication inédites. Le réseau télématique présente une nature profondément, constitutionnellement, interobjective.

\section{INTERNET: INTEROBJECTIVITÉ ET INTERTEXTUALITÉ}

Internet fonctionne donc comme système d'interconnexion entre objets de nature différente, et en même temps comme système d'interconnexion entre textes. En effet, la première dimension d'interobjectivité à prendre en considération est justement celle de la composition de l'intertexte.

À l'origine de la théorie des hypertextes, deux grands modèles semblent se dégager. L'un, moins intéressant selon nous, conçoit l'hypertexte comme extériorisation des structures associatives présentes dans la pensée humaine. L'autre, celui de la bibliothèque, postule la présence dans les textes de fils qui, secrètement, traversent les volumes, liant des pages d'œuvres différentes selon une logique intelligible quoique implicite, et accessible seulement au terme d'une enquête approfondie: une logique qui appartient à la culture comme telle plutôt qu'aux individus qui la produisent.

Autrement dit, à la base de la théorie des hypertextes, il y a entre autres l'hypothèse selon laquelle le système culturel est composé d'une collection de réalités textuelles produites au moyen d'opérations séparées et apparemment autonomes, mais dont le sens effectif dépend de la manière dont ces réalisations, à un niveau moins immédiatement visible, se lient entre elles, en donnant vie à un inextricable entrecroisement de renvois, allusions, associations, transformations, complémentarités et intégrations.

La théorie structuraliste, à laquelle est encore rattachée toute une sémiotique, soutient que l'identité profonde des différents objets composant le système dépend de leur position dans la structure générale, donc de l'ensemble de leurs liaisons avec les autres éléments de ce système. Plus radicale et plus explicite, la position de Saussure en vient à affirmer que la 
réalité de chaque composant particulier est uniquement relationnelle, c'est-à-dire que l'identité d'un composant n'est constituée de rien d'autre que du réseau de relations qui, en tant que telles, donnent vie au système.

Dans la perspective d'une étude des relations interobjectives, cela nous amène à une remarque fondamentale: nous saisissons comme des objets ce qui souvent devrait n'être saisi que comme des entités purement relationnelles. Ces relations sont des relations entre objets, mais aussi des relations entre objets et sujets (la conception greimassienne de l'objet de valeur est un très bon exemple du fait qu'on perçoit comme «objet» indépendant ce qui, en réalité, est une relation installée entre une entité étrangère et un sujet valorisant). La différence, par rapport à notre hypothèse, semblerait résider dans le fait qu' ici on se réfère aussi à des relations de nature opératoire, tandis qu'il s'agirait ailleurs de relations de nature purement cognitive. L'origine d'Internet pourrait coïncider avec le moment où a été conçue la possibilité de passer d'un modèle théorique - qui considérait le système culturel comme un réseau de textes - à un modèle précisément opératoire, où les connexions ont été rendues visibles, activables, dynamiquement praticables ${ }^{2}$.

Voyons donc comment la sémiotique a tenté de rendre compte aussi de la dimension opératoire du réseau intertextuel. On peut regretter qu'on ait jusqu'à maintenant trop peu étudié la manière dont les relations intertextuelles sont créées et activées, obligeant la sémiotique à accomplir rapidement une tâche importante et indispensable, rendue plus urgente encore par le développement fulgurant d'Internet. Dans l'immédiat, il faut reconnaître Claude Lévi-Strauss comme l'un des explorateurs les plus fervents de cette dimension. Sa façon de concevoir le système textuel (dans son cas, les récits mythiques des peuples sans écriture) comme un grand réseau, qui pose les entités sémiotiques comme des identités relationnelles, pressent de façon significative l'idée de l'immense toile d'araignée hypertextuelle déployée sur le monde, pour dessiner la logique des formes culturelles.
Il faut noter que sa recherche attribue aux textes en tant que tels une remarquable autonomie par rapport aux sujets qui en sont les auteurs. Le narrateur n'est pas du tout conscient des opérations qu'il est en train d'exécuter, et cela justement à cause de l'action des processus qui relient les récits entre eux. Même le faire des sujets peut ainsi être vu comme immergé dans le réseau des rapports interobjectifs.

La portée de la logique d'interconnexion textuelle paraît si forte à l'anthropologue français, et située à un niveau si différent de celui où s'établit la conscience des narrateurs, qu'il en est venu à énoncer la fameuse et fascinante assertion qui figure dans l' "Ouverture» du premier volume des Mythologiques:

Nous ne prétendons donc pas montrer comment les hommes pensent dans les mythes, mais comment les mythes se pensent dans les hommes, et à leur insu. ${ }^{3}$

Faut-il donc penser que des objets de valeur culturelle et sociale si importante peuvent être créés grâce à un jeu amplement interobjectif, plutôt que sous le contrôle d'un projet humain?

Cette prééminence des rapports interobjectifs sur l'instance du sujet, au moment crucial où le sens et les structures textuelles mêmes sont définis, parait décisive dans le cadre argumentatif qui est le nôtre. On pourrait d'ailleurs remarquer que cette perspective n'a pas de quoi étonner, dans cette époque où prédomine la sensation de vivre dans une réalité économique (et par conséquent politique) maîtrisée de façon déterminante par la force des choses. Mais la perspective lévi-straussienne est concomitante de ces nouveaux procédés d'interaction entre "objets", qui sont caractéristiques du réseau télématique (ce qui apporte quelques corrections, par exemple, aux thèses triomphalistes et un peu naïves de Lévy sur une «intelligence collective») ${ }^{4}$.

L'analyse de cette sorte d'interrelations entre objets pose nécessairement la question d'une définition du statut même du sujet. Que reste-t-il, en effet, à ce sujet, puisqu'il est dépouillé de la possibilité de contrôler la construction et le sens véritable de son propre discours? La sémiotique sociale, qui semble enfin sur 
le point de voir le jour, devra effectuer le passage d'une conception de genre encore trop subjective ${ }^{5}$ (qui tend à souligner le rôle d'artisan du sujet individuel et sa perception des faits de communication) à une conception beaucoup plus complexe, centrée sur des terrains d'interaction sociale, où le sujet qui agit est invariablement surpris et dépassé par ses interactants ${ }^{6}$.

\section{LES OBJETS REMPLACENT LES ÊTRES HUMAINS}

Il n'est pas banal de souligner que l'ancêtre de l'agent est d'abord l'usuel agenda sur lequel nous inscrivons les rendez-vous et les échéances quotidiennes. Il est tout à fait évident que l'agenda n'est pas comme tel du domaine de l'interobjectivité; cependant ses qualités fonctionnelles sont très remarquables, comme l'est son emplacement en tant qu'objet qui «interface» deux différentes positions occupées - dans le temps - par un même sujet. Il faut tout de suite noter que l'agenda est évidemment une réalité de nature textuelle.

Un regard à mon agenda personnel me permet de remarquer que les informations qui y sont inscrites ne sont pas toutes de même nature. Il est vrai que, dans la plupart des cas, il s'agit de purs éléments d'information, capables d'enregistrer échéances, horaires, personnes à contacter. Certains de ces engagements sont cependant entourés de parenthèses ou énergiquement soulignés: ce qui indique ma modalisation personnelle par rapport à eux (un «nonpouvoir" ou bien un "non-vouloir y prendre part» ou, vice versa, un «non-pouvoir ne pas y prendre part»). Et si l'agenda est d'abord conçu comme une représentation du temps linéaire (les heures s'écoulent, les jours, les semaines, etc.), on peut y introduire quelque procédé graphique pour rendre compte d'une autre dimension temporelle: celle qui correspond aux cas - pas si rares - de superposition temporelle. Si, par exemple, une leçon est fixée à la même heure qu'un conseil de faculté, le degré d'importance (ou encore de préférence) est marqué dans mon agenda par l'emploi de la couleur rouge, adoptée ici pour rendre bien visible une indication de nature modale, un «devoir» ou un «vouloir». Je constate aussi mon habitude de différencier, grâce à l'emploi d'une langue et d'un alphabet différents, des mots ou de petites phrases qui correspondent non pas à des informations mais plutôt à des recommandations que je m'adresse à moi-même («Rappelle-toi», «Très important», «Sois ponctuel», etc.).

$\mathrm{Si}$ la raison pour laquelle on ressent le besoin de noter dans son agenda les rendez-vous et les échéances est évidente, la raison pour laquelle on peut pareillement ressentir le besoin d'inscrire, et pour soimême, des estimations d'importance ou des jugements de prédilection n'est pas très différente. Nous nous reconnaissons évidemment comme des créatures imparfaites, distraites et inconstantes. L'agenda personnel témoigne de notre souci d'assurer une plus forte persistance dans le temps à nos attitudes modales: nos attitudes cognitives bien sûr, mais aussi nos attitudes à propos de ce que nous devons, ou voulons, ou bien avons le pouvoir de faire. En tant qu'intermédiaire installé entre deux différents «moi», l'agenda cherche à conformer le sujet postérieur au précédent: opération sûrement légitime, du moment qu'elle s'effectue sous le plein contrôle et aussi sous la responsabilité explicite du principal intéressé.

L'utilisation de l'agenda est sans aucun doute un premier pas vers l'attribution à un objet de tâches qui seraient spécifiques au sujet. Certaines de ces tâches concernent l'organisation et la gestion du temps, et pour celles-ci nous estimons en principe souhaitable une prise en charge toujours plus large de la part des objets. Par exemple, les agendas informatisés des personnes qui poursuivent des projets communs ont déjà l'habitude de se mettre en contact, d'échanger des informations, de trouver la meilleure solution pour une réunion conjointe qui ne se superpose à aucun autre engagement, et tout cela sans intervention humaine. Cet exemple nous montre comment, une fois que certaines tâches leur ont été attribuées, des objets peuvent aisément élargir leurs fonctions au niveau d'une collaboration entre objets. Nous sommes en présence, dans ces cas, de formes de délégation qui nous permettent d'être représentés par nos agents: et ceux-ci, dans cette perspective, développent des qualités fonctionnelles qui étaient déjà propres, entre autres, 
aux répondeurs téléphoniques, mais avec la capacité sans cesse accrue de remplacer les sujets humains.

Les bots agissent, en premier lieu, comme tout autre logiciel, sur la base d'instructions qui leur sont originellement données. Le degré de complexité et de sophistication de ces créatures logicielles est cependant très variable: certaines d'entre elles peuvent être douées de formes d'intelligence artificielle qui leur permettent d'adapter leurs modèles de comportement au moyen de processus d'«apprentissage». Et l' idée qui nous vient à l'esprit en premier lieu (elle a déjà été mise à l'épreuve à différentes occasions), c'est de faire en sorte que ces créatures apprennent en modelant leur apprentissage sur notre comportement. On comprend que cela s'avère très simple quand il s'agit de genres de comportement qui s'imposent par le biais de l'ordinateur, et on reconnaît que ce domaine se développe toujours davantage. Nos agents peuvent enregistrer et classer nos préférences dans les achats, nos intérêts dans les différents secteurs de l'information, les lieux de vacances qui attirent particulièrement notre attention, etc., de manière à pouvoir transposer ces données à de nouvelles activités. Tout ce qui permet de déceler quelque régularité est, en ce sens, susceptible de modélisation. De telles extrapolations peuvent ne pas être couronnées de succès, mais elles peuvent sûrement maximiser les probabilités que les agents parviennent à pointer le produit ou les vacances qui correspondent le mieux à nos désirs. Leur capacité d'action - leur pouvoir-faireest l'expression directe d'un savoir qui a pour objet notre vouloir.

Tout compte fait, cela parvient à renverser la direction normale de l'emploi expressif des objets. Couramment, on le sait bien, les objets (nous allons distinguer ce type d'objets en les appelant "objets-1") sont employés comme indicateurs d'une identité sociale: une identité qui, grâce à eux, pourra être lue par les autres sujets. Ici, au contraire, les objets (nous les appellerons «objets-2») agissent comme des lecteurs, enclins à cueillir tout indice de l'identité du sujet au service duquel ils se trouvent. Il est évident qu'en exécutant cette tâche, les agents (c'est-à-dire les objets-2) accorderont une attention toute spéciale aux éléments capables de les aider à saisir les aspects de notre personnalité qui peuvent le mieux être codifiés, donc à saisir justement les objets-1. Dans ce jeu d'objets qui "parlent» à d'autres objets, la définition de notre identité repose finalement sur un rapport interobjectif, quoique intégralement circonscrit dans ce qui fait notre personnalité. Il s'ensuit que les agents, dans la forme typique de raisonnement - programméqu'ils pratiquent («si... alors...»), ont une forte tendance à figer dans le temps nos attitudes et nos préférences.

\section{LES ROBOTS EN SOCIÉTÉ}

Si on considère la situation, on peut dire que ce processus est appelé à échapper bientôt au domaine du "personnel». Les bots sont des entités destinées à la communication comme aux échanges contractuels. Et le genre d'extrapolation qu'ils exercent sur nos indices de modalisation va fatalement tendre à se réaliser, tant du côté du «client» que du côté du «fournisseur». Dès aujourd'hui, par exemple, les supermarchés en ligne fournissent une aide aux opérations d'achat en suggérant au client régulier les produits qui figuraient déjà sur ses précédentes listes d'achats, mais qui, n'étant pas sur la liste actuelle, pourraient avoir fait l'objet d'un oubli: on offre donc la commodité de composer une nouvelle liste à partir d'un modèle de liste tiré des achats précédents. Cela peut réduire considérablement le temps nécessaire pour noter les produits à commander et l'attention pour se rappeler ce dont on a besoin. Il est bien évident que de tels outils - qui sans doute constituent une extension de notre ancien agenda personnel - visent par leur nature à reproduire nos précédents modèles d'achat. Il ne s'agit pas nécessairement d'une banale répétition du choix des mêmes produits, puisqu'un logiciel «débrouillard" sera sans doute en mesure de varier la liste en y introduisant de nouveaux produits: cependant, la logique qui se situe au-dessous des choix ne pourra pas être modifiée, car il s'agit des critères fondamentaux de goût du sujet en fonction desquels le bot opère. 
Prenons l'exemple d'un «agent personnel», de type conservateur, qui tombe sur un «bot-vendeur» qui a tendance, pour des raisons pratiques (nous nous gardons de parler de gentillesse), à maintenir dans le temps une logique de sélection constante.

L'interaction entre objets donne rapidement lieu à une perverse obstination en faveur des habitudes, sinon à une véritable pulsion de répétition. Si les robots, qui planifient nos vacances, ou les réfrigérateurs et les fours connectés, qui organisent notre quotidien, tendent inévitablement à figer, à répéter - réduisant ainsi fortement toute forme d'expérimentation innovatrice ou même de hasard -, n'arriveront-ils pas à interdire toute possibilité de changement, de découverte, de trouvailles inattendues?

Rendre le monde plus contrôlable revient à réduire les possibilités d'entropie, mais nous sommes ici devant une situation inverse par rapport à l'habitude: «les choses» ne présentent plus une tendance naturelle à l'entropie, mais au contraire à l'excès de redondance, à l'inertie.

La logique du marché aspire d'ailleurs à de plus vastes conquêtes, car il s'agit, par sa nature et depuis toujours, d'une logique extensionniste. Pourquoi un client $\mathrm{X}$ se ravitaille-t-il chez un marchand uniquement pour les produits en boîte et, par exemple, chez un autre pour les surgelés? Admettons que la philosophie du premier marchand ne puisse pas lui permettre d'utiliser un bot, fort en sociosémiotique, pour déduire les préférences en matière de surgelés à partir de celles qu'il a observées dans les achats de produits en boîte. Ce marchand disposera certainement alors d'un «socio-bot» capable d'opérer une analyse de groupe à partir des observations nombreuses qu'il aura collectées sur les listes d'achats de tous ses clients.

Il lui sera possible de dire au client $\mathrm{X}$ que ceux qui, parmi ses clients, suivent habituellement sa logique d'achat de produits en boîte apprécient aussi certains produits surgelés. Ce client $\mathrm{X}$ pourra ainsi se sentir rassuré et encouragé à l'achat.

Ce phénomène se produit aujourd'hui dans certaines librairies en ligne, où l'on constate qu'un système automatisé nous propose avec acuité de nouveaux livres susceptibles de nous intéresser, puisqu'ils ont déjà intéressé d'autres lecteurs qui, au terme d'analyse, ont montré qu'ils partageaient dans l'ensemble nos choix de lecture. Le «biblio-bot" passe ainsi du dispositif de l'extension rapporté à chaque sujet particulier à celui de l'extension entre sujets, entre sujets qu'il suppose partager un même modèle (culturel, dans ce cas). On comprend que ce genre d'automates est en mesure d'établir des coalitions avec d'autres automates, à la suite bien sûr d'accords entre les êtres humains, étendant transversalement, pour ainsi dire, la base de construction des modèles de prévision de nos tendances, de manière à permettre par exemple à un «tourist-bot» de nous suggérer des vacances susceptibles de nous plaire, d'après nos préférences dans le domaine des aliments, des livres et des vêtements ${ }^{7}$. Il faut noter que nous ne sommes pas en train de pointer de sinistres opérations d'espionnage de notre "vie privée», mais de relever simplement l'utilisation de données acquises dans des lieux considérés comme "publics» (mais le rapport entre «public» et "privé» est lui aussi en pleine transformation conceptuelle).

Il ne s'agit pas ici d'aborder les problèmes complexes que tout cela peut entraîner en regard de la protection de la «vie privée», mais il peut être intéressant d'approfondir quelques-unes des raisons qui peuvent déterminer une résistance de notre part. Ce qui nous inquiète dans ce phénomène, c'est en fait la façon dont se resserre autour de nous cette chaîne d'objets qui nous lient à leurs agendas, et qui, en définitive, nous interprètent. D'un côté, nous craignons qu'un geste accompli spontanément - un acte presque atypique - soit interprété comme partie intégrante de notre identité: on ne voudrait pas, par exemple, que l'achat en passant d'un numéro de Playboy nous fasse passer pour des mordus de ce genre de publications. D'un autre côté, nous ne voulons pas non plus que ce qui nous est habituel (même s'il n'y a rien qui y soit répréhensible) soit fixé dans une définition qui, de toute façon, nous paraitra étroite, inadéquate et impropre. Finalement, le point capital du conflit entre 
nous et les «choses» pourrait se résumer ainsi: les «choses» peuvent être classées, définies, positionnées, mais pas nous.

D'ailleurs, la qualité principale qu'on reconnaît au «Sujet» n'est pas justement d'être inachevé: ce qui le conduit inlassablement à se donner une définition de lui-même déplacée par rapport à sa façon d'être; une définition concevable uniquement pour représenter une entité, projetée ailleurs, que non sans raison nous avons l'habitude d'appeler "Objet" ${ }^{8}$. Cette opération produit une certaine dose d'imprévisible, d'inconnu, nous présentant comme étrangers à nos propres yeux. Les rapports intersubjectifs jouent, en effet, sur ces facteurs leurs meilleures cartes. La séduction par exemple, en tant que triomphe de l'intersubjectivité, se réalise comme subtil croisement de projets, d'absences et de fantômes - simulation d'une identification de l'autre à ce qu'il n'est pas ${ }^{9}$.

Il ne s'agit ici que de simples rapports intersubjectifs «à deux». Le tableau du faire sémiotique opérant sur le plan collectif, installé lui aussi à l'intérieur de cet espace fondamental de tension qui sépare les buts d'un programme de ses données de départ, sera évidemment d'autant plus complexe. La dimension sociale multiplie évidemment les possibilités de composition relationnelle entre les projets individuels, en les embrouillant dans une série complexe de modalisations, d'inclusions, mais aussi de virtualités, chacune édifiée sur la base de l'autre.

On pourrait dire que l'intertextualité est la sociabilité perçue au niveau textuel. Le rapport entre les textes dans cette perspective efface leur nature proprement "objective», puisque l'interaction entre les objets sémiotiques, au lieu de les fixer dans le temps, les ouvre à des identités et à des possibilités de significations toujours nouvelles.

La notion lévi-straussienne de «transformation" est à ce propos spécialement remarquable. Elle vient à l'encontre de l'idée d'un changement d'état. Il est possible, selon le fondateur de l'anthropologie structurale, de penser les transformations sans avoir recours aux notions d'antériorité ou de postériorité: autrement dit, il est possible de concevoir la transformation comme un état. Un «état de transformation" - suivant un modèle de dérivation topologique indirecte - correspond à la possibilité de penser une correspondance entre les éléments agencés à l'intérieur de différents systèmes de référence, de sorte que, en connaissant les coordonnées d'un élément dans un certain système, il soit possible de préciser les règles qui permettent d'obtenir les coordonnées de l'élément correspondant dans un autre système. Autrement dit, deux systèmes «en transformation », bien qu'ils soient différents, divergent selon une logique précise de traduction et de déformation réciproque. L'espace social correspond à ce champ de correspondances et de torsions qui placent les groupes, les individus et les structures sémiotiques dans un état de transformation réciproque.

Est-ce qu'on pourrait alors vraiment s'attendre à ce que ces créatures logicielles raffinées, destinées à nous tenir compagnie dans un proche avenir, soient en mesure d'accéder au rang de sujets-en-société? Même dans le domaine économique et commercial, ces créatures devront opérer dans un monde dominé par les valeurs signifiées, plus que par les fonctionnalités. Le marché sera encore davantage consacré à la production d'entités intangibles et de mondes imaginaires, soucieux de vendre des «expériences" inédites plutôt que des "produits». Il faut donc sans doute s'attendre - puisque cela est techniquement possible - à des robots qui nous proposeront des expériences qu'on n'aurait jamais imaginées: des réfrigérateurs nous proposant de nouvelles sortes de boissons et des fours nous offrant une cuisine créative; des bots aux suggestions imprévues et aux conseils innovateurs: des agents spécialisés, fournisseurs d'imaginaire, capables de nous surprendre en nous conduisant dans leurs propres rêves. Cela peut aisément arriver grâce à un enchaînement d'échanges entre les objets (et par conséquent, indirectement et secrètement, entre les sujets): échanges de projets d'action, parcours de valorisation, structures qui interprètent les événements et qui décodent les entités signifiantes. Les bots échangeront ainsi, entre les 
humains, les pathémisations, les expériences, les aspirations, les modalisations et tout autre état virtuel. Cela est possible non pas parce qu'on peut penser que de tels instruments sont en mesure d'échapper à leur nature de "choses", mais parce que l'interaction entre les objets «intelligents» ouvre sans doute des perspectives imprévues. Dans cette interaction, il est permis de croire que ce qui est "donné», "connu», "constaté", grâce à la relation avec plusieurs autres "données", parviendra à produire l'inattendu - c'est-àdire une composante réputée essentielle dans l'interaction humaine.

Les objets «intelligents» pourront ainsi dépasser la logique unipolaire de la permanence maintenue sur l'axe antécédent/conséquent, et parviendront enfin à se reconnaitre eux-mêmes comme des objets appartenant à un circuit social, comme une réalité qui n'est plus centrée sur des simulacres de sujets autonomes, mais déplacée sur les formes de leurs appartenances et de leurs interactions.

Ils seront là où aucune action ne possède en soi son sens accompli et -comme l'écrit Bruno Latourlà où tout prend le caractère d'une médiation. Les acteurs de la scène sociale se présentent en effet essentiellement comme des médiateurs, et aucun d'eux n'est jamais précisément en position ni de cause ni de conséquence par rapport à ses partenaires ${ }^{10}$.

\section{N O TES}

1. A. Leonard, Bots. The Origin of New Species, San Francisco, Hard Wired, 1997, p. 10. C'est nous qui traduisons.

2. Cf. à ce propos l'espace agi dont parlent G. Bettetini, B. Gasparini et N. Vittadini (Gli spazi dell'ipertesto, Milano, Bompiani, 1999).

3. C. Lévi-Strauss, Le Cru et le Cuit, Paris, Plon, 1964, p. 20.

4. P. Lévy, L'Intelligence collective. Pour une anthropologie du cyberspace, Paris, La Découverte, 1994.

5. Cf. G. Ferraro, La Pubblicità nell'era di Internet, Roma, Meltemi, 1999, en particulier les pages 69-71.

6. Cf. B. Latour, «Une sociologie sans objet? Remarques sur

l'interobjectivité", Sociologie du travail, vol. 4, 1994, p. 587-607.

7. Il est encore plus facile de "nous acheter" chez un marchand de profils personnels, construits peut-être grâce à un réseau de robots bavards...

8. Je me réfère évidemment à l'objet de valeur de la théorie greimassienne.

9. À propos du dispositif de la séduction, cf. G. Ferraro, op. cit., p. 79ssq.

10. Cf. B. Latour, op. cit., p. 601. 